\title{
High-resolution Detector for Neutron Diffraction and Quantification of Subsurface Residual Stress
}

\author{
Stuart R. Miller ${ }^{1, a^{*}}$, Matthew S.J.Marshall ${ }^{1, b}$, Megan Wart ${ }^{1, c}$, \\ Pijush Bhattacharya $^{1, d}$, Stephen Puplampu ${ }^{2, e}$, Matthew Seals ${ }^{2, f}$, \\ Dayakar Penumadu $^{2,9}$, Rick Riedel ${ }^{3, \mathrm{~h}}$, and Vivek V. Nagarkar ${ }^{1, \mathrm{i}}$ \\ ${ }^{1}$ Radiation Monitoring Devices, Inc., Watertown, MA 02472, USA \\ ${ }^{2}$ University of Tennessee, Knoxville, TN 37996, USA \\ ${ }^{3}$ Neutron Scattering Science Division, Oak Ridge National Laboratory, PO Box 2008, Building \\ 7962, Oak Ridge, TN 37831-6393, USA \\ a*smiller@rmdinc.com, ${ }^{\mathrm{b}} \mathrm{mmarshall@rmdinc.com,}{ }^{\mathrm{C}} \mathrm{mwart} @$ rmdinc.com, \\ dpbhattacharya@rmdinc.com, e spuplamp@vols.utk.edu, ${ }^{\mathrm{f}} \mathrm{mseals2@vols.utk.edu,}$ \\ gdpenumad@utk.edu, hriedelra@ornl.gov, ivnagarkar@rmdinc.com
}

Keywords: Neutron Scattering, Neutron Diffraction, Anger Camera, Residual Stress Measurement, Lithium Sodium Iodide

\begin{abstract}
We have developed a new high-resolution large-area detector for neutron diffraction imaging, specifically for the measurement of subsurface residual stress in engine components. Neutron diffraction typically requires monochromatic thermal or cold neutrons, neutron flux at the detector is orders of magnitude lower than for standard neutron radiography. Therefore the detection efficiency for incident neutrons must be maximized in order to reduce acquisition times. Here we use the high absorption cross section of LNI ( $\left.{ }^{6} \mathrm{Li}_{\mathrm{x}} \mathrm{Na}_{1-\mathrm{x}} \mathrm{I}: \mathrm{Eu}, \mathrm{Tl}\right)$ scintillators coupled to an Anger camera consisting of an array of silicon photomultiplier (SiPM) detectors. The LNI scintillator developed at RMD is derived from the well-known NaI scintillator and comes in two formats for this application, a vapor-deposited film and a crystal sliced into 1-2 $\mathrm{mm}$ thick layers for imaging. In either case, $95 \%$ enriched ${ }^{6} \mathrm{Li}$ was utilized. LNI crystal slices have demonstrated high light yield, for example a $1 \mathrm{~mm}$ thick sample with $10 \% \mathrm{Li}$ produced a 40,900 photons/MeV gamma response and a neutron response with gamma equivalent energy (GEE) of $3.76 \mathrm{MeV}$, indicating 153,800 photons/neutron. In general higher Li content decreases the light yield but increases the GEE up to $4.8 \mathrm{MeV}$, which is approximately the theoretical maximum. Use of spatially resolved detectors for diffraction signals from single and polycrystalline materials is gaining strong interest from the neutron community, particularly from advanced light source users who benefit from plenty of photons to work with. Since diffraction signals from polycrystalline materials are inherently weak, the use of this approach at neutron facilities, which typically provide a low flux of neutrons in the thermal or cold energy ranges, poses unique problems. This paper presents aspects of utilizing high-resolution and high efficiency neutron detectors for obtaining important engineering measurements such as residual stress considering the target materials of interest. We present for the first time measurements associated with diffraction signals captured using an Anger camera with high spatial resolution for an example scattering polycrystalline powder appropriate for the incident mono-energetic cold neutrons at the CG1 (Cold Guide Hall) beamline of the High Flux Isotope Reactor at the Oak Ridge National Laboratory.
\end{abstract}




\section{Introduction}

Neutron diffraction is an important tool for crystallography to provide information about the structure of materials. While these measurements are typically done at a high flux beamline from a reactor or spallation source, the scattered neutrons incident at the detector plane are typically quite low in quantity due to the fact that they are scattered from a target and also monochromatized. Thus the detector requirements for neutron diffraction imaging place a premium on detection efficiency in order to capture as many of the incident neutrons as possible.

The Anger Camera, developed by the detector group at the Oak Ridge National Laboratory, coupled with a highly efficient scintillator, provides the required detection efficiency [1,2]. In addition it has the advantage of a large active measurement area and high spatial resolution for detecting diffracted/scattered thermal neutrons. Implementation of pulse shape (in addition to pulse height) discrimination further enhances the ability to detect diffracted neutrons with enhanced signal to noise ratio.

Here we are developing a neutron imaging detector specifically for the quantification of residual stress in metallic engine components. The goal is to make this possible with a neutron generator in a laboratory environment. This makes detector requirements even more critical due to a lower flux of neutrons incident on the detector.

A key component of our detector development is the scintillator that captures neutrons and converts them efficiently to optical photons for detection by the Anger camera. RMD is developing the LNI $\left({ }^{6} \mathrm{Li}_{\mathrm{x}} \mathrm{Na}_{1-\mathrm{x}} \mathrm{I}: \mathrm{Eu}, \mathrm{Tl}\right)$ scintillator that combines the high absorption of ${ }^{6} \mathrm{Li}$ with the well-known NaI scintillator; LNI technology is patented under US 9,417,343 [3].

\section{Anger Camera}

The anger camera is the detector of choice for this application due to its high detection efficiency as well as the capability to use PSD and PHD for neutron/gamma discrimination. The Anger camera is a variant within the CCD imaging family that allows for fast read-out of large multianode photo-multiplier tubes. Instead of approaching the issue of readout by establishing a channel for each anode; the Anger camera reduces the entire output of the PMT down to four channels allowing for fast data acquisition over the entire photo-sensitive area in real-time [4,5]. The output from an array of PMT's is injected into a resistive network where Anger logic is used to determine the location of incident particles on the scintillator.

The Anger camera used here has an active area of $15 \times 15 \mathrm{~cm}^{2}$ and consists of a $16 \mathrm{x} 16$ array of SiPM's.

\section{LNI Scintillator Development}

The development of the LNI scintillator has two formats, including vapor-deposited films and crystal slices. The advantage of the film format is the columnar structure that produces enhanced spatial resolution for a given thickness of scintillator. Fig. 1 shows the SEM images of an LNI film showing the columnar structure that allows the scintillation light to be channeled to the detector, allowing thicker films to be used while maintaining high
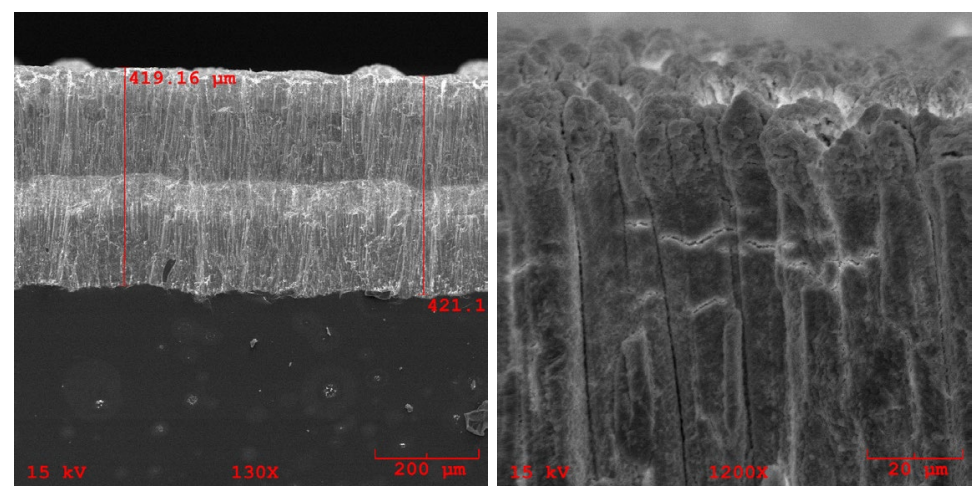

Figure 1: (Left) SEM image of a $420 \mu \mathrm{m}$ thick LNI film. (Right) Close up of the columns at the top of the film. Note that some degradation of the film structure has occurred due to exposure to room atmosphere. 
spatial resolution. When coupled to the Anger camera a $400 \mu \mathrm{m}$ thick film has demonstrated the very high spatial resolution of $350 \mu \mathrm{m}$, as shown in Fig. 2. This is the highest observed with the Anger camera.

Another approach being explored is to grow the LNI in crystal format and then cut the crystal into thin slices that are 1-4 mm thick, as shown in Fig. 3. While this approach can't provide the high resolution observed from films, it allows thicker layers of scintillators to be used to provide the needed stopping power for neutron scatter imaging. Due to the Anger logic method of single photon detection, monolithic crystal slices provide a good combination of efficiency and spatial resolution. The challenge with this approach is
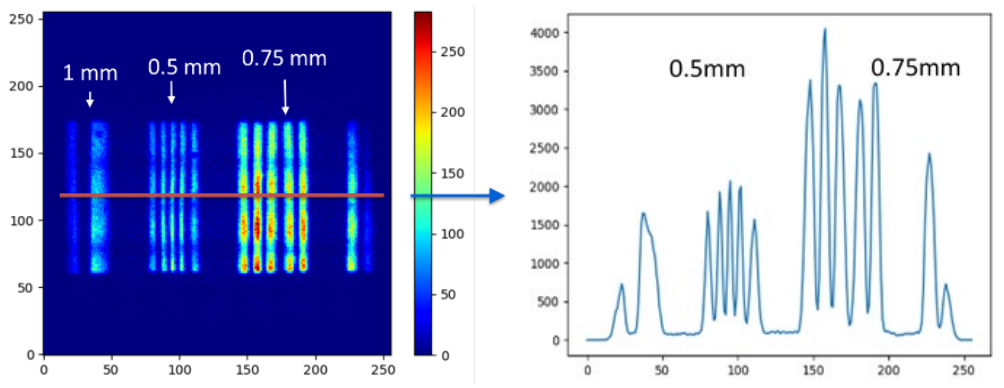

Figure 4: (Left) Resolution mask image acquired using a 4"x4", 500 um thick, LNI film to an SiPM Anger camera. (Right) Line profile demonstrating a high degree of modulation even for a $0.5 \mathrm{~mm}$ pattern. Estimated resolution is $\sim 350$ um.
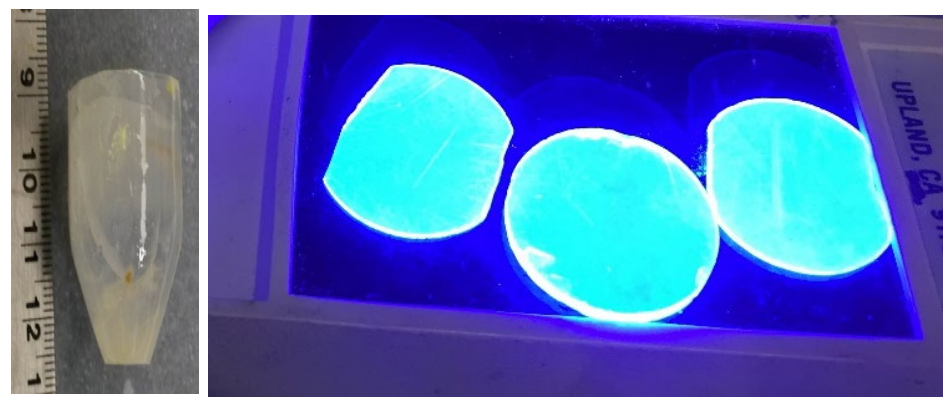

Figure 2: (Left) An LNI crystal with $20 \%{ }^{6}$ Li enriched to $95 \%$. (Right) Several crystal slices cut from the crystal at left, with 1, 3 and $2 \mathrm{~mm}$ thicknesses, viewed with UV excitation. to grow crystals large enough for imaging, and cutting thin slices without cracking. Currently we are developing the methods to achieve this.

\section{Neutron Response}

The neutron response was measured by coupling the LNI crystal slices to a 3" PMT (Hamamatsu R6233-100 SBA). The 20\% LNI provided a very high gamma-equivalent energy (GEE) of $4.8 \mathrm{MeV}$, as shown in Fig. 4, which is on par with the theoretical Q-value for ${ }^{6} \mathrm{Li}$ conversion. This sample produced 15,000 photons/MeV gamma light yield and 72,200 photons/neutron. A sample with $10 \% \quad{ }^{6} \mathrm{Li}$ however produced 40,900 photons/MeV gamma response and a neutron response with GEE of $3.76 \mathrm{MeV}$, indicating 153,800 photons/neutron. This is on par with and

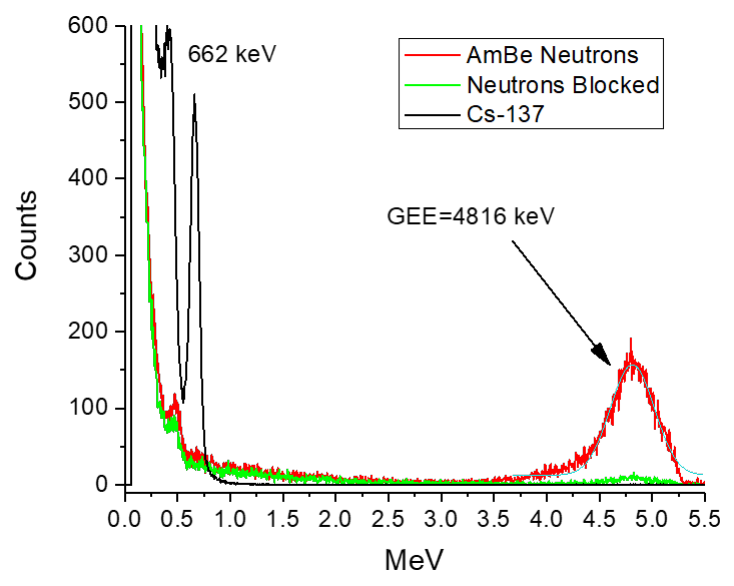

Figure 3: Neutron response of a $2 \mathrm{~mm}$ thick LNI crystal sample with $20 \%{ }^{6} \mathrm{Li}$. Gamma equivalent energy is $4.8 \mathrm{MeV}$ which is the theoretical maximum. slightly exceeding previously reported results [6].

The neutron response of LNI films has been previously reported [7] and a GEE of $4.3 \mathrm{MeV}$ was observed from a $375 \mu \mathrm{m}$ thick film. 
Both crystals and films provide excellent gamma-neutron discrimination [1,2], which is particularly important for neutron scattering measurements and the Anger camera has the capability to take advantage of this to remove gamma events and increase signal-noise ratio in the images.

\section{Neutron Absorption Efficiency}

The neutron absorption efficiencies were measured for LNI films of various thicknesses. The measurements were taken with the beam on and aperture open and beam on and aperture closed and compared to a ${ }^{3} \mathrm{He}$ detector standard. The measured efficiencies as a function of LNI thickness are shown in $\mathbf{5}$, and the results are commensurate with the theoretical values with $50 \%$ ${ }^{6} \mathrm{Li}$ films.

\section{Diffraction Imaging}

Diffraction measurements were demonstrated by coupling a $450 \mu \mathrm{m}$ thick LNI film to the Anger camera. The diffraction line from a germanium rod was imaged with a $300 \mathrm{~s}$ acquisition time with the detector $65 \mathrm{~mm}$ from the sample, as shown in Fig. 6. Imaging was performed at the HFIR CG-1A beamline. This shows that an LNI film with about 50\% efficiency can produce high qualiy diffraction images, however the image acquisition times are still long. We are developing thicker LNI films that are also brighter, and expect to improve on this result.

\section{Summary}

We have developed a new large-area detector for neutron diffraction imaging based on an Anger camera coupled to an LNI scintillator with the following attributes:

- High sensitivity, up to $70 \%$

- High spatial resolution, $350 \mu \mathrm{m}, 1.4 \mathrm{lp} / \mathrm{mm}$

- Neutron/Gamma discrimination with PHD

- The LNI scintillator can be in film or crystal format.

The LNI scintillator coupled to the Anger camera provides the highest possible combination of detection efficiency and spatial resolution. This detector is currently under development for use in the imaging of diffraction peaks from various materials. By mapping diffraction peak changes it is then possible to determine the stress state of the objects of interest. Future developments are planned to optimize the detector to measure and map the residual stress in 
engine components and to make it possible to perform these measurements in a laboratory setting.

\section{Acknowledgements}

This work is currently being supported by Department of Defense Grant N6833517C0250.

\section{References}

[1] H.O. Anger, Review of Scientific Instruments, 29(1) (1959) 27.

https://doi.org/10.1063/1.1715998

[2] R.A.Riedel, et. al., Design and performance of a large area neutron sensitive anger camera, NIM in Physics Research A 794 (2015) 224-233. https://doi.org/10.1016/j.nima.2015.05.026

[3] V.Nagarkar, H. Bhandari, and O. Ovechkina, United States Patent "Neutron Detector and Fabrication Method Thereof, US 9,417,343 B1, August 16, 2016.

[4] P. D. Olcott, J. A. Talcott, C. S. Levin, F. Habte, and A. M. K. Foudray, "Compact readout electronics for position sensitive photomultiplier tubes," IEEE Transactions on Nuclear Science, vol. 52, no. 1, pp. 21-27, 2005. https://doi.org/10.1109/TNS.2004.843134

[5] S. Siegel, R. W. Silverman, S. Yiping, and S. R. Cherry, "Simple charge division readouts for imaging scintillator arrays using a multi-channel PMT," IEEE Transactions on Nuclear Science, vol. 43, no. 3, pp. 1634-1641, 1996. https://doi.org/10.1109/23.507162

[6] Nagarkar, V., Ovechkina, E., Bhandari, H., Miller, S., Marton, Zs., Glodo, J., SoundaraPandian, L., Mengesha, W., Gerling, M., Brubaker, E., 2013. Lithium alkali halides - new thermal neutron detectors with $n-\gamma$ discrimination, Nuclear Science Symposium and Medical Imaging Conference (NSS/MIC), 2013 IEEE.

[7] Marshall, M.S.J., M.J. More, H.B. Bhandari, R.A. Riedel, S. Waterman, J. Crespi, P. Nickerson, S. Miller, V.V. Nagarkar, "Novel Neutron Detector Material: Microcolumnar LixNa1-xI:Eu," in IEEE Transactions on Nuclear Science, vol. 64, no. 11, pp. 2878-2882, Nov. 2017. doi: 10.1109/TNS.2017.2762859. https://doi.org/10.1109/TNS.2017.2762859 